Purdue University

Purdue e-Pubs

2005

\title{
Analysis and Optimization of the Thermal Performance of Microchannel Heat Sinks
}

Dong Liu

University of Houston

S V. Garimella

Purdue University, sureshg@purdue.edu

Follow this and additional works at: http://docs.lib.purdue.edu/coolingpubs

Liu, Dong and Garimella, S V., "Analysis and Optimization of the Thermal Performance of Microchannel Heat Sinks" (2005). CTRC Research Publications. Paper 59.

http://dx.doi.org/10.1108/09615530510571921

This document has been made available through Purdue e-Pubs, a service of the Purdue University Libraries. Please contact epubs@purdue.edu for additional information. 


\title{
Analysis and Optimization of the Thermal Performance of Microchannel Heat Sinks ${ }^{\dagger}$
}

\author{
Dong Liu and Suresh V. Garimella \\ Cooling Technologies Research Center \\ School of Mechanical Engineering \\ Purdue University, West Lafayette, IN 47907-2088 USA
}

\begin{abstract}
A number of modeling approaches of increasing levels of complexity for the analysis of convective heat transfer in microchannels are presented and compared. A detailed computational fluid dynamics (CFD) model is used to obtain baseline results against which the different approximate approaches are compared. These include a 1-D resistance model, a fin approach, two fin-liquid coupled models, and a porous medium approach, all of which are amenable to closed-form solutions for the temperature field. The good agreement between the exact and approximate methods indicates that with carefully chosen assumptions, these analytical results can lead to adequate descriptions of the thermal performance, while allowing easier manipulation of microchannel geometries for the purpose of optimization. Practical optimization procedures are developed to minimize the overall thermal resistance of microchannel heat sinks, using each of the five approaches.
\end{abstract}

Keywords: microchannels; optimization; heat sink; electronics cooling

\footnotetext{
${ }^{\dagger}$ Submitted for publication in International Journal of Numerical Methods for Heat and Fluid Flow, February 2003, and in revised form, May 2003.

$\$$ Corresponding author: (765)494-5621, sureshg@ecn.purdue.edu
} 


\section{Nomenclature}

$A_{c}$ microchannel cross-sectional area

$A_{f} \quad$ fin cross-sectional area

$A_{s} \quad$ area of heat sink

$C_{p}$ specific heat

$D_{h}$ hydraulic diameter

fRe friction constant

$h$ heat transfer coefficient

$H$ height of heat sink

$H_{c}$ microchannel depth

$k$ thermal conductivity

$L \quad$ length of heat sink

$\dot{m}$ mass flow rate

$n \quad$ number of microchannels

$\mathrm{Nu}$ Nusselt number

$P$ pressure

$q$ " heat flux

$q$ heat removal rate

$Q \quad$ volume flow rate

$R \quad$ thermal resistance

Re Reynolds number

$t$ substrate thickness
$T$ fin temperature

$T_{b} \quad$ temperature at the base of the fin

$T_{f} \quad$ fluid temperature

$u_{m}$ mean flow velocity

$W \quad$ width of heat sink

$w_{c}$ microchannel width

$w_{w}$ fin thickness

Greek Symbols

$\alpha \quad$ aspect ratio of microchannels

$\eta_{f} \quad$ fin efficiency

$\mu \quad$ dynamic viscosity

$\theta$ thermal resistance

$\rho$ density of fluid

$\Delta p$ pressure drop

$\Delta T$ temperature difference

Subscripts and Superscripts

c channel

$f \quad$ fluid

$i \quad$ inlet

$s \quad$ solid fin

$w \quad$ wall 


\section{Introduction}

The potential for handling ultra-high heat fluxes has spurred intensive research into microchannel heat sinks (Tuckerman and Pease, 1981; Weisberg and Bau, 1992; Sobhan and Garimella, 2001). For implementation in practical designs, the convective heat transfer in microchannels must be analyzed in conjunction with the choice and optimization of the heat sink dimensions to ensure the required thermal performance. Design procedures are also needed to minimize the overall thermal resistance.

The focus of this paper is to present a comprehensive discussion and comparison of five different (approximate) analytical models of increasing sophistication, which offer closed-form solutions for single phase convective heat transfer in microchannels. A general CFD model is first set up to obtain an "exact" solution. Details of the approximate models and the assumptions involved are then presented along with a comparison of the thermal resistance predictions from these models. Optimization of the thermal performance of microchannel heat sinks is then discussed.

\section{Description of the Problem}

The microchannel heat sink under consideration is depicted in Fig. 1. Materials for fabrication may include conductive materials such as copper and aluminum for modular heat sinks, or silicon if the microchannels are to be integrated into the chip. For conservative estimates of thermal performance, the lid (top plate) may be to be insulated. The width of individual microchannels and intervening fins $\left(w_{c}+w_{w}\right)$ is typically small compared to the overall heat sink dimension $W$, and numerous channels are accommodated in parallel flow paths. 
Continuum equations for conservation of mass, momentum and energy, respectively, for the convective heat transfer in microchannel heat sinks can be written as (Fedorov and Viskanta, 2000; Toh, et al., 2002):

$$
\begin{aligned}
& \nabla \cdot(\rho \vec{V})=0 \\
& \vec{V} \cdot \nabla(\rho \vec{V})=-\nabla P+\nabla \cdot(\mu \nabla \vec{V}) \\
& \vec{V} \cdot \nabla\left(\rho C_{p} T\right)=\nabla \cdot\left(k_{f} \nabla T\right) \text { for the fluid } \\
& \nabla \cdot\left(k_{s} \nabla T_{s}\right)=0 \text { for the fin }
\end{aligned}
$$

This set of equations assumes steady-state conditions for incompressible, laminar flow, with radiation heat transfer neglected. With an appropriate set of boundary conditions, these equations provide a complete description of the conjugate heat transfer problem in microchannels.

\section{Take in Figure 1}

\section{CFD Model}

A numerical model was formulated to solve for the three-dimensional heat transfer in microchannels using the commercial CFD software package, FLUENT (Fluent, 1998). The simulation was performed for three different sets of dimensions as listed in Table I. These three cases are chosen to simulate experiments in the literature (Tuckerman and Pease, 1981) that have often been used for validating numerical studies (Weisberg and Bau, 1992; Toh, et al., 2002; Ryu, et al., 2002).

\section{Take in Figure 2}

The computational domain, chosen from symmetry considerations, is shown in Fig. 2. The top surface is adiabatic and the left and right sides are designated symmetric boundary conditions. A uniform heat flux is applied at the bottom surface. In the present work, water is used as the 
working fluid $\left(\rho=997 \mathrm{~kg} / \mathrm{m}^{3}, C_{p}=4179 \mathrm{~J} / \mathrm{kgK}, \mu=0.000855 \mathrm{~kg} / \mathrm{ms}\right.$, and $k_{f}=0.613 \mathrm{~W} / \mathrm{mK}$ evaluated at $27^{\circ} \mathrm{C}$ ), and silicon is used as the heat sink substrate material with $k_{s}=148 \mathrm{~W} / \mathrm{mK}$.

\section{$\underline{\text { Take in Table I }}$}

In the numerical solution, the convective terms were discretized using a first-order upwind scheme for all equations. The entire computational domain was discretized using a $500 \times 60 \times 14$ $(x-y-z)$ grid. To verify the grid independence of the convective heat transfer results, three different meshes were used in the fluid part of the domain: $20 \times 5,30 \times 7$, and $50 \times 15$. The thermal resistance changed by $3.4 \%$ from the first to the second mesh, and only by $0.3 \%$ upon further refinement to the third grid. Hence $30 \times 7$ grids were used in the fluid domain for the results in this work.

The agreement between the experimental and predicted values of thermal resistance in Table I validates the use of the numerical predictions as a baseline against which to compare the approximate approaches considered in this work.

The numerical results may also be used to shed light on the appropriate boundary conditions for the problem under consideration. For instance, it is often assumed in microchannel heat sink analyses that the axial conduction in both the solid fin and the fluid may be neglected. Using the numerical results for Case 1 as an example, the axial conduction through the fin and fluid were found to account for $0.3 \%$ and $0.2 \%$ of the total heat input at the base of the heat sink, respectively. Thus the assumption of negligible axial conduction appears valid for heat transfer in the silicon microchannels considered.

\section{Take in Figure 3}

Two alternative boundary conditions have been commonly used at the base of the fin in microchannel analyses (Zhao and Lu, 2002; Samalam, 1989; Sabry, 2001): 


$$
-\left.k_{s} \frac{\partial T}{\partial y}\right|_{y=0}=q^{\prime \prime}
$$

or

$$
-\left.k_{s} \frac{d T}{d y}\right|_{y=0}=\frac{w_{c}+w_{w}}{w_{w}} q^{\prime \prime}
$$

in which Eq. (5) implies that the imposed heat flows evenly into the fluid via the bottom of the microchannel and into the fin via the base of the fin, while Eq. (6) implies that all the heat from the base travels up the base of the fin. Clearly, neither of these two extreme cases represents the actual situation correctly. The computed heat flux in the substrate in the immediate vicinity of the fin base is shown in Fig. 3 for Case 1. The heat fluxes into the fluid and the fin are 55.5 $\mathrm{W} / \mathrm{cm}^{2}$ and $333 \mathrm{~W} / \mathrm{cm}^{2}$, respectively. Hence, the error associated with employing Eqs. (5) and (6) as the boundary condition at the base of the fin would be $50 \%$ and $24 \%$, respectively. A reasonably accurate alternative for the boundary condition could be developed as follows:

$$
q=h\left(\frac{w_{c}}{2} L\right)\left(T_{b}-\bar{T}_{f}\right)+h\left(H_{c} L\right) \eta_{f}\left(T_{b}-\bar{T}_{f}\right)
$$

Hence, the ratio of the heat dissipated through the vertical sides of the fin to that flowing through the bottom surface of the microchannel into the fluid is $2 \eta_{f} H_{c} / w_{c}$, or $2 \eta_{f} \alpha$. This leads to a more reasonable boundary condition at the base of the fin:

$$
-\left.k_{s} \frac{d T}{d y}\right|_{y=0}=\frac{2 \eta_{f} \alpha}{2 \eta_{f} \alpha+1} \frac{w_{c}+w_{w}}{w_{w}} q^{\prime \prime}
$$

This condition results in a heat flux of $366 \mathrm{~W} / \mathrm{cm}^{2}$ through the base of the fin, which is within $10 \%$ of the computed exact value of $333 \mathrm{~W} / \mathrm{cm}^{2}$.

In light of this discussion, Eq. (8) is imposed as the thermal boundary condition at the base of the fin for all the five approximate models developed in this work. 


\section{Approximate Analytical Models}

In view of the complexity and computational expense of a full CFD approach for predicting convective heat transfer in microchannel heat sinks, especially in searching for optimal configurations under practical design constraints, simplified modeling approaches are sought. The goal is to account for the important physics, even if some of the details may need to be sacrificed. Five approximate analytical models (Zhao and Lu, 2002; Samalam, 1989; Sabry, 2001; Kim and Kim, 1999) are discussed, along with the associated optimization procedures needed to minimize the thermal resistance. The focus in this discussion is on the development of a set of thermal resistance formulae that can be used for comparison between models, as well as for optimization of microchannel heat sinks.

As shown in Fig. 1, for the problem under considertaion, the fluid flows parallel to the $\mathrm{x}$-axis. The bottom surface of the heat sink is exposed to a constant heat flux. The top surface remains adiabatic.

The overall thermal resistance is defined as

$$
R_{o}=\frac{\Delta T_{\max }}{q^{\prime \prime} A_{s}}
$$

in which $\Delta T_{\max }=\left(T_{w, o^{-}} T_{f, i}\right)$ is the maximum temperature rise in the heat sink, i.e. the temperature difference between the peak temperature in the heat sink at the outlet $\left(T_{w, o}\right)$ and the fluid inlet temperature $\left(T_{f, i}\right)$. Since the thermal resistance due to substrate conduction is simply

$$
R_{\text {cond }}=\frac{t}{k_{s}(L W)}
$$

the thermal resistance $R$ calculated in following models will not include this term:

$$
R=R_{o}-R_{\text {cond }}
$$


The following assumptions are made for the most simplified analysis:

1. Steady-state flow and heat transfer

2. Incompressible, laminar flow

3. Negligible radiation heat transfer

4. Constant fluid properties

5. Fully developed conditions (hydrodynamic and thermal)

6. Negligible axial heat conduction in the substrate and the fluid

7. Averaged convective heat transfer coefficient $h$ for the cross section.

In the approximate analyses considered, this set of assumptions is progressively relaxed.

\section{Model 1 - 1D Resistance Analysis}

\section{$\underline{\text { Take in Figure } 4}$}

In addition to making assumptions 1 to 7 above, the temperature is assumed uniform over any cross section in the simplest of the models.

For fully developed flow under a constant heat flux, the temperature profile within the microchannel in the axial direction is shown in Fig. 4. The three components of the heat transfer process are:

$$
\begin{aligned}
& q_{\text {cond }}=k_{s} A_{s} \frac{T_{w, o}-T_{b, o}}{t} \\
& q_{\text {conv }}=h A_{f}\left(T_{b}-\bar{T}_{f}\right) \\
& q_{c a l}=\rho Q C_{p}\left(T_{f, o}-T_{f, i}\right)
\end{aligned}
$$

The overall thermal resistance can thus be divided into three components: 


$$
\begin{aligned}
\mathrm{R}_{\mathrm{o}} & =\frac{\Delta T_{\max }}{q^{\prime \prime}(L W)}=\frac{1}{q^{\prime \prime}(L W)}\left[\left(T_{w, o}-T_{f, i}\right)\right] \\
& =R_{\text {cond }}+R_{\text {conv }}+R_{c a l}
\end{aligned}
$$

in which the three resistances may be determined as follows:

1. Conductive thermal resistance

$$
R_{\text {cond }}=\frac{t}{k_{s}(L W)}
$$

2. Convective thermal resistance

$$
R_{c o n v}=\frac{1}{n h L\left(2 \eta_{f} H_{c}+w_{c}\right)}
$$

with fin efficiency $\eta_{f}=\frac{\tanh \left(m H_{c}\right)}{m H_{c}}$.

3. Caloric thermal resistance:

$$
R_{c a l}=\frac{1}{\rho_{f} Q C_{p}}
$$

\section{Model 2 - Fin Analysis}

In this model, assumptions 1 to 7 above are adopted, and the fluid temperature profile is considered one-dimensional (averaged over y-z cross section), $T_{f}=T_{f}(x)$. The temperature distribution in the solid fin is then:

$$
\frac{d^{2} T}{d y^{2}}=\frac{2 h}{k_{s} w_{w}}\left(T-T_{f}(x)\right)
$$

with boundary conditions:

$$
-\left.k_{s} \frac{d T}{d y}\right|_{y=0}=\frac{2 \eta_{f} \alpha}{2 \eta_{f} \alpha+1} \frac{w_{c}+w_{w}}{w_{w}} q^{\prime \prime}
$$




$$
\left.\frac{d T}{d y}\right|_{y=H_{c}}=0
$$

It follows that

$$
T(x, y)=T_{f}(x)+\frac{1}{m} \frac{q^{\prime \prime}}{k_{s}} \frac{2 \eta_{f} \alpha}{2 \eta_{f} \alpha+1} \frac{w_{c}+w_{w}}{w_{w}} \frac{\cosh \left\{m\left(H_{c}-y\right)\right\}}{\sinh \left(m H_{c}\right)}
$$

where $m=\left(2 h / k_{s} w_{w}\right)^{1 / 2}$.

The fluid temperature $T_{f}(x)$ can be obtained from an energy balance:

$$
\dot{m} C_{p} \frac{d T_{f}(x)}{d x}=q^{\prime \prime} \cdot\left(w_{c}+w_{w}\right)
$$

with $T_{f}(x=0)=T_{0}$. The bulk fluid temperature is then:

$$
T_{f}(x)=T_{0}+\frac{q^{\prime \prime}\left(w_{c}+w_{w}\right)}{\rho_{f} C_{p} u_{m} H_{c} w_{c}} x
$$

and Eq. (22) can be rewritten as:

$$
T(x, y)=T_{0}+\frac{1}{m} \frac{q^{\prime \prime}}{k_{s}} \frac{2 \eta_{f} \alpha}{2 \eta_{f} \alpha+1} \frac{w_{c}+w_{w}}{w_{w}} \frac{\cosh \left\{m\left(H_{c}-y\right)\right\}}{\sinh \left(m H_{c}\right)}+\frac{q^{\prime \prime}\left(w_{c}+w_{w}\right)}{\rho_{f} C_{p} u_{m} H_{c} w_{c}} x
$$

The thermal resistance is thus:

$$
R=\frac{\Delta T}{q^{\prime \prime}(L W)}=\frac{T(L, 0)-T_{0}}{q^{\prime \prime}(L W)}=\frac{1}{m} \frac{1}{k_{s}} \frac{2 \eta_{f} \alpha}{2 \eta_{f} \alpha+1} \frac{w_{c}+w_{w}}{w_{w}} \frac{\cosh \left(m H_{c}\right)}{\sinh \left(m H_{c}\right)} \frac{1}{(L W)}+\frac{\left(w_{c}+w_{w}\right)}{\rho_{f} C_{p} u_{m} H_{c} w_{c}} \frac{1}{W}
$$

\section{Model 3 - Fin-Fluid Coupled Approach I}

Following the same line of reasoning as in the fin analysis (Model 2) and adopting assumptions 1 to 7 above, but averaging the fluid temperature only in the $\mathrm{z}$ direction (Samalam, 1989), the energy equation in the fin can be written as:

$$
\frac{\partial^{2} T}{\partial y^{2}}=\frac{2 h}{k_{s} w_{w}}\left(T-T_{f}(x, y)\right)
$$


with

$$
\begin{gathered}
-\left.k_{s} \frac{\partial T}{\partial y}\right|_{y=0}=\frac{2 \eta_{f} \alpha}{2 \eta_{f} \alpha+1} \frac{w_{c}+w_{w}}{w_{w}} q^{\prime \prime}=J \\
\left.\frac{\partial T}{\partial y}\right|_{y=H_{c}}=0
\end{gathered}
$$

The energy balance in the fluid is represented by:

$$
\rho_{f} C_{p} u_{m} w_{c} \frac{\partial T_{f}}{\partial x}=2 h\left(T-T_{f}(x, y)\right)
$$

and it is assumed that $T_{f}(x=0, y)=0$. Substituting $h=N u k_{f} / D_{h}$ into Eq. (30) yields

$$
\frac{1}{2} \rho_{f} C_{p} u_{m} w_{c} D_{h} \frac{\partial T_{f}}{\partial x}+k_{f} N u T_{f}=k_{f} N u T
$$

Defining $X=x / \mathrm{a}$ and $Y=y / a$ where $a=\frac{\rho_{f} C_{p} u_{m} w_{c} D_{h}}{2 k_{f} N u}$, the solution to Eq. (31) can be written as

$$
T_{f}(X, Y)=\int_{0}^{X} T\left(X^{\prime}, Y\right) e^{-\left(X-X^{\prime}\right)} d X^{\prime}
$$

Hence, Eq. (27) can then be transformed to

$$
\frac{\partial^{2} T(X, Y)}{\partial Y^{2}}=\beta\left\{T(X, Y)-\int_{0}^{X} T\left(X^{\prime}, Y\right) e^{-\left(X-X^{\prime}\right)} d X^{\prime}\right\}
$$

in which

$$
\begin{aligned}
& \beta=\frac{a^{2}}{\lambda^{2}} \\
& \lambda^{2}=\frac{k_{s} w_{w} D_{h}}{2 k_{f} N u}
\end{aligned}
$$

Solving Eq. (33) by Laplace transforms,

$$
\frac{\partial^{2} f(Y, s)}{\partial Y^{2}}=\gamma f \quad\left(\gamma=\frac{\beta s}{s+1}\right)
$$

The boundary conditions in Eqs. (28) and (29) become 


$$
\begin{aligned}
& -\left.k_{s} \frac{\partial f}{\partial Y}\right|_{Y=0}=\frac{J a}{s} \\
& -\left.k_{s} \frac{\partial f}{\partial Y}\right|_{Y=\tilde{H}_{c}}=0
\end{aligned}
$$

in which $\tilde{H}_{c}=H_{c} / a$, and $J$ is defined in Eq. (28). The solution to this system of equations is

$$
f(s)=\frac{J a}{k_{s} s \sqrt{\gamma}} \frac{\cosh \left(\sqrt{\gamma}\left(Y-\tilde{H}_{c}\right)\right)}{\sinh \left(\sqrt{\gamma} \tilde{H}_{c}\right)}
$$

The inverse Laplace transform yields the temperature:

$$
\begin{aligned}
T(X, Y) & =L^{-1}[f(s, Y)] \\
& =\frac{J a}{k_{s} \tilde{H}_{c} \beta}\left\{(1+X)+\frac{\beta}{2}\left(Y-\tilde{H}_{c}\right)^{2}-\frac{\beta \tilde{H}_{c}^{2}}{6}+2 \sum_{n=1}^{\infty}(-1)^{n} \frac{\left(s_{n}+1\right)^{2}}{s_{n}} \cos \left[\frac{n \pi\left(Y-\tilde{H}_{c}\right)}{\tilde{H}_{c}}\right] e^{s_{n} X}\right\}
\end{aligned}
$$

in which

$$
s_{n}=\frac{-n^{2} \pi^{2} / \tilde{H}_{c}^{2}}{\beta+n^{2} \pi^{2} / \tilde{H}_{c}^{2}} .
$$

This is a rapidly converging infinite series for which the first three terms adequately represent the thermal resistance:

$$
R=\frac{\Delta T}{q^{\prime \prime}(L W)}=\frac{T(L, 0)-T_{0}}{q^{\prime \prime}(L W)}=\frac{J a}{k_{s} \tilde{H}_{c} \beta}\left[(1+L / a)+\frac{1}{3} \beta \tilde{H}_{c}^{2}\right] \frac{1}{(L W)}
$$

\section{Model 4 - Fin-Fluid Coupled Approach II}

In this model, assumptions 1 to 7 above are again adopted, except that axial conduction in the fin is not neglected (Sabry, 2001). The governing equations in the solid fin and liquid, respectively, are therefore:

$$
\nabla^{2} T(x, y, z)=0
$$




$$
\nabla \cdot\left(\rho_{f} C_{p} \vec{V} T_{f}(x, y, z)\right)=k_{f} \nabla^{2} T_{f}(x, y, z)
$$

At the fin-fluid interface, the condition is

$$
-k_{s} \frac{\partial T_{s}}{\partial z}=-k_{f} \frac{\partial T_{f}}{\partial z}=h\left(T_{i}-\bar{T}_{f}\right)
$$

in which the averaged local fluid temperature is

$$
\bar{T}_{f}(x)=\int_{0}^{w_{c} / 2} v T_{f} d z /\left(u_{m} w_{c} / 2\right)
$$

with $u_{m}=\int_{0}^{w_{c} / 2} v d z / w_{c} / 2$. Along with Eq. (28), the following boundary conditions apply:

$$
\left.\frac{\partial T}{\partial y}\right|_{y=H_{c}}=\left.\frac{\partial T}{\partial x}\right|_{x=0}=\left.\frac{\partial T}{\partial x}\right|_{x=L}=\left.\frac{\partial T}{\partial z}\right|_{z=-\frac{w_{w}}{2}}=\left.\frac{\partial T_{f}}{\partial z}\right|_{z=\frac{w_{c}}{2}}=0
$$

Integrating Eq. (42) over $\mathrm{z}$ from $-w_{w} / 2$ to 0 , the fin temperature varies as

$$
\frac{w_{w}}{2}\left(\frac{\partial^{2}}{\partial x^{2}}+\frac{\partial^{2}}{\partial y^{2}}\right) \bar{T}+\left.\frac{\partial \bar{T}}{\partial z}\right|_{-\frac{w_{w}}{2}}=0
$$

in which $\bar{T}=\int_{-\frac{w_{w}}{2}}^{0} T d z /\left(w_{w} / 2\right)$. Combining Eqs. (44) and (46),

$$
k_{s} \frac{w_{w}}{2}\left(\frac{\partial^{2}}{\partial x^{2}}+\frac{\partial^{2}}{\partial y^{2}}\right) \bar{T}-h\left(T_{i}-\bar{T}_{f}\right)=0
$$

Assuming $T_{i}=\bar{T}$, since $B i=h\left(w_{w} / 2\right) / k_{s}<<1$, Eq. (48) becomes

$$
k_{s} \frac{w_{w}}{2}\left(\frac{\partial^{2}}{\partial x^{2}}+\frac{\partial^{2}}{\partial y^{2}}\right) \bar{T}-h\left(\bar{T}-\bar{T}_{f}\right)=0
$$

If the axial conduction term $\frac{\partial^{2} \bar{T}}{\partial x^{2}}$ were neglected, Eq. (49) would reduce to Eq. (27).

Since fully developed conditions are assumed and axial conduction in the fluid is neglected, Eq. (43) may be integrated over $\mathrm{z}$ from 0 to $w_{c} / 2$ to yield 


$$
\frac{\partial}{\partial x} \int_{0}^{w_{c} / 2} u T_{f} d z=\left.\frac{k_{f}}{\rho_{f} C_{p}} \frac{\partial T_{f}}{\partial z}\right|_{0} ^{\frac{w_{c}}{2}}
$$

Using the boundary condition in Eq. (44), this reduces to

$$
u_{m} \frac{w_{c}}{2} \frac{\partial}{\partial x} \bar{T}_{f}+\frac{1}{\rho_{f} C_{p}} h\left(\bar{T}_{f}-\bar{T}\right)=0
$$

The following dimensionless variables are introduced:

$$
X=x / L, Y=y / H_{c} \text { and } T=\frac{\bar{T}-T_{0}}{\Delta T_{c}}
$$

in which

$$
\Delta T_{c}=\frac{2 \eta_{f} \alpha}{2 \eta_{f} \alpha+1} \frac{\left(w_{c}+w_{w}\right) / 2}{h H_{c}} q^{\prime \prime}
$$

The system of equations above can be cast in dimensionless terms:

$$
\begin{aligned}
& \left(A^{2} \frac{\partial^{2}}{\partial X^{2}}+\frac{\partial^{2}}{\partial Y^{2}}\right) T-\left(m H_{c}\right)^{2}\left(T-T_{f}\right)=0 \\
& \frac{\partial T_{f}}{\partial X}+S\left(T_{f}-T\right)=0 \\
& \left.\frac{\partial T}{\partial Y}\right|_{Y=0}=-\left(m H_{c}\right)^{2} \\
& \left.\frac{\partial T_{s}}{\partial X}\right|_{X=1}=\left.\frac{\partial T_{s}}{\partial Y}\right|_{Y=0}=\left.\frac{\partial T_{s}}{\partial Y}\right|_{Y=1}=0 \\
& \left.T_{f}\right|_{X=0}=0
\end{aligned}
$$

where $A=H_{c} / L$ and the modified Stanton number (Sabry, 2001) is given by $S=h L /\left(\rho_{f} C_{p} u_{m} \frac{w_{c}}{2}\right)$

Employing similar techniques as adopted for Model 3, the fin temperature is obtained as 


$$
T(X, Y)=m H_{c} \frac{\cosh \left(m H_{c}(1-Y)\right)}{\sinh \left(m H_{c}\right)}+\sum_{n=0}^{\infty} \cos (n \pi Y) f_{n}(X)
$$

in which the first term in the infinite series provides results of acceptable accuracy $(<5 \%$ deviation from the complete series):

$$
f_{0}(X)=S X+\sum_{i=1}^{2}\left(\frac{C_{0 i}}{w_{0 i}}\right) e^{w_{0 i} X}+C_{03}
$$

with

$$
\begin{aligned}
& w_{01}=-\frac{S}{2}+\sqrt{\left(\frac{S}{2}\right)^{2}+\left(\frac{m H_{c}}{A}\right)^{2}} \\
& w_{02}=-\frac{S}{2}-\sqrt{\left(\frac{S}{2}\right)^{2}+\left(\frac{m H_{c}}{A}\right)^{2}} \\
& C_{01}=-S \frac{\left(e^{w_{02}}-1\right)}{\left(e^{w_{02}}-e^{w_{01}}\right)} \\
& C_{02}=-S \frac{\left(e^{w_{01}}-1\right)}{\left(e^{w_{02}}-e^{w_{01}}\right)} \\
& C_{03}=\left(\frac{S A}{m H_{c}}\right)^{2}
\end{aligned}
$$

The thermal resistance is thus obtained as

$$
\begin{aligned}
R & =\frac{\Delta T}{q^{\prime \prime}(L W)}=\frac{T(1,0)-T_{0}}{q^{\prime \prime}(L W)} \Delta T_{c} \\
& =\frac{2 \eta_{f} \alpha}{2 \eta_{f} \alpha+1} \frac{w_{c}+w_{w}}{2 h H_{c}}\left[m H_{c} \frac{\cosh \left(m H_{c}\right)}{\sinh \left(m H_{c}\right)}+S+\sum_{i=1}^{2}\left(\frac{C_{0 i}}{w_{0 i}}\right) e^{w_{0 i}}+C_{03}\right] \frac{1}{(L W)}
\end{aligned}
$$

In most practical cases, $\left(m H_{c} / A\right)>>S / 2$, and Eq. (61) reduces to

$$
R=\frac{2 \eta_{f} \alpha}{2 \eta_{f} \alpha+1} \frac{w_{c}+w_{w}}{2 h H_{c}}\left[m H_{c} \frac{\cosh \left(m H_{c}\right)}{\sinh \left(m H_{c}\right)}+S+\left(\frac{S A}{m H_{c}}\right)^{2}\right] \frac{1}{(L W)}
$$




\section{Model 5 - Porous Medium Approach}

The convective heat transfer process in microchannels can also be treated as being similar to that in a fluid-saturated porous medium, with the extended Darcy equation used for fluid flow and a volume-averaged two-equation model used for heat transfer, as demonstrated in Vafai and Tien (1981).

Following the analysis of Kim and Kim (1999), a two-equation model can be employed to obtain the volume-averaged properties over a representative elementary volume for the solid region and the fluid region separately. The momentum equation and boundary conditions are

$$
\begin{gathered}
-\frac{d}{d x}\langle p\rangle_{f}+\mu_{f} \frac{d^{2}}{d y^{2}}\langle u\rangle_{f}-\frac{\mu_{f}}{K} \varepsilon\langle u\rangle_{f}=0 \\
\langle u\rangle_{f}=0 \text { at } y=0, H_{c}
\end{gathered}
$$

where $\langle u\rangle_{f}$ is the volume-averaged velocity, $\varepsilon=w_{c} /\left(w_{c}+w_{w}\right)$ is the porosity, and $K=\varepsilon w_{c}{ }^{2} / 12$ is the permeability. Equations (63) and (64) may be written as

$$
\begin{aligned}
& U=D a \frac{d^{2} U}{d Y^{2}}-P \\
& U=0 \text { at } Y=0,1
\end{aligned}
$$

using the dimensionless parameters

$$
U=\frac{\langle u\rangle_{f}}{u_{m}}, D a=\frac{K}{\varepsilon H^{2}}=\frac{1}{12 \alpha_{s}^{2}}, Y=\frac{y}{H}, P=\frac{K}{\varepsilon \mu_{f} u_{m}} \frac{d\langle p\rangle_{f}}{d x} .
$$

The solution to the momentum equation is then

$$
U=P\left\{\cosh \left(\sqrt{\frac{1}{D a} Y}\right)+\frac{1-\cosh \left(\sqrt{\frac{1}{D a}}\right)}{\sinh \left(\sqrt{\frac{1}{D a}}\right)} \sinh \left(\sqrt{\frac{1}{D a} Y}\right)-1\right\}
$$

The volume-averaged energy equations for the fin and fluid, respectively, are: 


$$
\begin{aligned}
& k_{s e} \frac{\partial^{2}\langle T\rangle}{\partial y^{2}}=h a\left(\langle T\rangle-\langle T\rangle_{f}\right) \\
& \varepsilon \rho_{f} C_{p}\langle u\rangle_{f} \frac{\partial\langle T\rangle_{f}}{\partial x}=h a\left(\langle T\rangle-\langle T\rangle_{f}\right)+k_{f e} \frac{\partial^{2}\langle T\rangle_{f}}{\partial y^{2}}
\end{aligned}
$$

with boundary conditions

$$
\begin{aligned}
& \langle T\rangle=\langle T\rangle_{f}=T_{w} \text { at } y=0 \\
& \frac{\partial\langle T\rangle}{\partial y}=\frac{\partial\langle T\rangle_{f}}{\partial y}=0 \text { at } y=H_{c}
\end{aligned}
$$

in which $a$ is the wetted area per unit volume, $h$ the local heat transfer coefficient defined as the ratio of the interfacial heat flux to the solid-fluid temperature difference, and $k_{s e}$ and $k_{f e}$ the effective conductivities of the solid and fluid, defined as $k_{s e}=(1-\varepsilon) k_{s}, k_{f e}=\varepsilon k_{f}$

For fully developed flow under constant heat flux, it is known that

$$
\frac{\partial\langle T\rangle_{f}}{\partial x}=\frac{\partial\langle T\rangle}{\partial x}=\frac{d T_{w}}{d x}=\text { constant }
$$

and

$$
q^{\prime \prime}=\varepsilon \rho_{f} C_{p} u_{m} H \frac{\partial\langle T\rangle_{f}}{\partial x}
$$

The energy equations (68) and (69) and boundary conditions can thus be written in dimensionless form as

$$
\begin{aligned}
& \frac{d^{2} \theta}{d Y^{2}}=D\left(\theta-\theta_{f}\right) \\
& U=D\left(\theta-\theta_{f}\right)+C \frac{d^{2} \theta_{f}}{d Y^{2}}
\end{aligned}
$$

with

$$
\theta=\theta_{f}=0 \quad \text { at } Y=0
$$




$$
\frac{d \theta}{d Y}=\frac{d \theta_{f}}{d Y}=0 \text { at } Y=1
$$

in which

$$
\begin{aligned}
& C=\frac{\varepsilon k_{f}}{(1-\varepsilon) k_{s}}, D=\frac{h a H^{2}}{(1-\varepsilon) k_{s}}, \\
& \theta=\frac{\langle T\rangle-T_{w}}{\frac{q^{\prime \prime} H}{(1-\varepsilon) k_{s}}} \text {, and } \theta_{f}=\frac{\langle T\rangle_{f}-T_{w}}{\frac{q^{\prime \prime} H}{(1-\varepsilon) k_{s}}} .
\end{aligned}
$$

Substituting the solution obtained for velocity, Eq. (74) and (75) can be solved to give

$$
\begin{gathered}
\theta_{f}=\frac{P}{1+C}\left[-\frac{1}{2} Y^{2}+C_{1} Y+C_{2}-C_{3} \times \cosh \left(\sqrt{\frac{D(1+C)}{C} Y}\right)-C_{4} \sinh \left(\sqrt{\frac{D(1+C)}{C} Y}\right)+C_{5} \times\right. \\
\left.\left\{\cosh \left(\sqrt{\frac{1}{D a}} Y\right)+\frac{1-\cosh \left(\sqrt{\frac{1}{D a}}\right)}{\sinh \left(\sqrt{\frac{1}{D a}}\right)} \sinh \left(\sqrt{\frac{1}{D a}} Y\right)\right\}\right] \\
\left.\theta=P\left[D a\left\{\cosh \left(\sqrt{\frac{1}{D a}} Y\right)+\frac{1-\cosh \left(\sqrt{\frac{1}{D a}}\right)}{\sinh \left(\sqrt{\frac{1}{D a}}\right)} \sinh \left(\sqrt{\frac{1}{D a}} Y\right)\right\}-\frac{1}{2} Y^{2}+C_{1} Y-D a\right]-C \theta_{f}\right] \\
(79)
\end{gathered}
$$

in which

$$
\begin{aligned}
& N_{1}=D(1+C) \sqrt{\frac{1}{D a}\left\{1-\cosh \left(\sqrt{\frac{1}{D a}}\right)\right\}} \\
& N_{2}=\frac{C}{D a} \sqrt{\frac{D(1+C)}{C}} \sinh \left(\sqrt{\frac{1}{D a}}\right) \sinh \left(\sqrt{\frac{D(1+C)}{C}}\right) \\
& C_{1}=1-\frac{\sqrt{D a}\left(\cosh \left(\sqrt{\frac{1}{D a}}\right)-1\right)}{\sinh \left(\sqrt{\frac{1}{D a}}\right)}
\end{aligned}
$$




$$
\begin{aligned}
& C_{2}=-D a+\frac{1}{D(1+C)} \\
& C_{3}=\frac{C}{D a D(1+C) D_{1}} \\
& C_{4}=\frac{N_{1}+N_{2}}{D(1+C) \sqrt{\frac{D(1+C)}{C}} \cosh \left(\sqrt{\left.\frac{D(1+C)}{C} Y\right) \sinh \left(\sqrt{\frac{1}{D a}}\right) D_{1}}\right.} \\
& C_{5}=D a-\frac{1}{D_{1}}
\end{aligned}
$$

Finally, the thermal resistance can be obtained as

$$
R=\frac{12 K}{\rho_{f} C_{p} w_{c}^{3} \alpha W \varepsilon^{2} u_{m}}-\frac{\theta_{f, b} H_{c}}{(1-\varepsilon) k_{s} L W}
$$

in which $\theta_{f, b}$ is the bulk mean fluid temperature, defined as

$$
\theta_{f, b}=\int_{0}^{1} U \theta_{f} d Y / \int_{0}^{1} U d Y
$$

Key features of the five approximate models discussed above, including the assumptions, governing equations and resistance formulae developed, are summarized in Table II.

\section{Assessment of the Approximate Models}

For the microchannel parameters listed in Table I, thermal resistances were computed with Fluent as well as from the five approximate models. The results are shown in Table III. It can be seen that all the approximate models would provide acceptable predictions for the thermal resistance of the microchannel heat sink, with the maximum deviation being $7.8 \%$. Models 2 through 5 are more complex to apply than Model 1, and involve the solution of the differential governing equations. In spite of its simplicity, Model 1 appears to adequately represent the physics of the heat transfer problem, and is recommended for use in the design and optimization 
of practical microchannel heat sinks.

\section{$\underline{\text { Take in Table III }}$}

It may be noted that in Model 2, the fluid temperature is considered to be only a function of the $x$-coordinate, and the fin temperature is solved in a truly 1-D manner. The thermal resistance expression from Model 2 is therefore identical to that from Model 1. Also, in Models 3 and 4, since 2-D temperature fields are considered in both the fin and the fluid, the new terms $H_{c} /\left(3 n k_{s} w_{w} L\right)$ and $\left(1 /\left(\rho_{f} C_{p} Q\right)^{2}\right)\left(n k_{s} H_{c} w_{w} / L\right)$ appear, in addition to the other terms in the simpler Models 1 and 2. The difference between Models 3 and 4 is that the axial conduction term appears explicitly in the fin equation of Model 4, while it is neglected in Model 3.

In the calculations above, expressions for Nusselt number $N u$ and the friction constant $f R e$ are needed for computing the convective heat transfer coefficient $h$ and the average velocity $u_{m}$ in the microchannel. In all five approximate models discussed in the present work, the flow is assumed to be thermally and hydrodynamically fully developed. Hence the following relations are used in terms of microchannel aspect ratios (Incropera and DeWitt, 1996; Shah and London, 1978):

$$
\begin{aligned}
& N u_{f d}=8.235\left(1-1.883 \alpha+3.767 \alpha^{2}-5.814 \alpha^{3}+5.361 \alpha^{4}-2 \alpha^{5}\right) \\
& (f \operatorname{Re})_{f d}=96\left(1-1.3553 / \alpha+1.9467 / \alpha^{2}-1.7012 / \alpha^{3}+0.9564 / \alpha^{4}-0.2537 / \alpha^{5}\right)
\end{aligned}
$$

However, the fully developed assumption is not always valid, especially for microchannels with the larger hydraulic diameters and short lengths. With hydrodynamic and thermal lengths defined as $L^{+}=L /\left(D_{h} \operatorname{Re}\right)$ and $L^{*}=L /\left(D_{h} \operatorname{Re} \operatorname{Pr}\right)$, the following relations (Samalam, 1989; Harms, et al., 1999) could be employed instead of Eqs. (81) and (82):

$$
N u=3.35\left(L^{*}\right)^{-0.13} \alpha^{0.12} \operatorname{Pr}^{-0.038}, 0.013 \leq L^{*}<0.1
$$




$$
\begin{aligned}
& N u=1.87\left(L^{*}\right)^{-0.30} \alpha^{0.056} \operatorname{Pr}^{-0.036}, 0.0005 \leq L^{*}<0.013 \\
& f_{\text {app }} \operatorname{Re}=\left[\left\{\frac{3.2}{\left(L^{+}\right)^{0.57}}\right\}^{2}+(f \operatorname{Re})_{f d}^{2}\right]^{1 / 2}, L^{+}<0.05
\end{aligned}
$$

In the present calculations, $L / D_{h} \approx 100$ with moderate Reynolds numbers, so that the hydrodynamically fully developed condition is satisfied. For the working fluid in this study (water, Prandtl number 5.8), Nusselt numbers calculated from Eqs. (81) and (83) are listed in Table IV. The deviation between the two sets of results is within $6 \%$, and therefore the assumption of thermally fully developed conditions is acceptable. In general, developing thermal effects should be carefully considered before fully developed conditions are assumed.

\section{$\underline{\text { Take in Table IV }}$}

\section{Optimization}

The optimization of microchannel heat sink design can be motivated using the thermal resistance approach in Model 1. As indicated in Eq. (18), $R_{\text {cal }}$ is inversely proportional to the mass flow rate. When the pressure head along the microchannel length is prescribed as the constraint, $R_{c a l}$ will decrease as $w_{c}$ increases when $H_{c}$ reaches the maximum allowable value. However, the convective heat transfer coefficient $h$ will increase when $D_{h}$ decreases, leading to a reduction in $R_{\text {conv }}$, as shown by Eq. (17). The heat transfer from the substrate through the fins will also be enhanced if the fin efficiency increases, which requires a larger fin thickness $w_{w}$. However, the increase in $w_{w}$ will reduce the number of microchannel/fin pairs in a heat sink for a prescribed heat sink size. Due to these competing factors, there exists an optimal microchannel dimension that minimizes the overall thermal resistance. 
In order to optimize the thermal performance of a microchannel heat sink, the following variables must be specified from implementation constraints:

1. Thermal conductivity of the bulk material used to construct the heat sink $\left(k_{S}\right)$;

2. Overall dimension of the heat sink ( $L$ and $W$ from the size of the chip, $H_{c}$ and $t$ from fabrication and structural considerations);

3. Properties of the coolant $\left(\rho_{f}, \mu, k_{f}, C_{p}\right)$; and

4. Allowable pressure head $(\Delta P)$.

To illustrate the procedure, the example considered uses water as the working fluid to cool a chip with $L=W=1 \mathrm{~cm}$ and a given pressure head of $\Delta P=60 \mathrm{kPa}$. The heat load is $100 \mathrm{~W} / \mathrm{cm}^{2}$. The microchannel heat sink is to be made of silicon with $t=100 \mu m$ and $H_{c}=400 \mu m$. The fluid properties are evaluated at $27^{\circ} \mathrm{C}$. The optimization process involves finding the optimal microchannel geometry (channel width $w_{c}$, fin thickness $w_{w}$ and aspect ratio $\alpha=H_{c} / w_{c}$ ) that will minimize thermal resistance.

Solutions to the following equations would yield the optimum:

$$
\begin{gathered}
\frac{\partial R}{\partial w_{c}}=0 \\
\frac{\partial R}{\partial w_{w}}=0
\end{gathered}
$$

In this work, the optimization computations were performed using the commercial solver MATLAB (MathWorks, 2001). The optimized results derived from the five approximate models are listed in Table V. The optimal thermal resistance values reported from the five models agree to within $10 \%$. It may also be noted that the minimum thermal resistance is always attained at the largest allowable aspect ratio. In practical designs, the aspect ratio would be determined by the limits on the microchannel depth and the substrate thickness. 


\section{$\underline{\text { Take in Table V }}$}

\section{Conclusions}

Five approximate analytical models for predicting the convective heat transfer in microchannel heat sinks are presented and compared. Closed-form solutions from these models are compared to full CFD simulation and experimental results, and the efficacy of the different models assessed. Optimization procedures are discussed for minimizing the thermal resistance of the heat sinks. The results obtained demonstrate that the models developed offer sufficiently accurate predictions for practical designs, while at the same time being quite straightforward to use.

\section{Acknowledgements}

The authors acknowledge the financial support from members of the Cooling Technologies Research Center (http://widget.ecn.purdue.edu/ CTRC), a National Science Foundation Industry/University Cooperative Research Center at Purdue University.

\section{References}

Fedorov, A. G. and Viskanta, R. (2000), "Three-dimensional conjugate heat transfer in the microchannel heat sink for electronic packaging", International Journal of Heat Mass Transfer, Vol. 43, no. 3, pp. 399-415.

Fluent User's Guide, (1998), Fluent Inc. Lebanon, New Hampshire.

Harms, T. M., Kazmierczak, M. J. and Gerner, F. M. (1999), "Developing convective heat transfer in deep rectangular microchannels", International Journal of Heat Fluid Flow, Vol. 20, no. 2, pp. 149-157.

Incropera, F. P. and DeWitt, D. P. (1996), Fundamentals of Heat and Mass Transfer, John Wiley \& Sons, N. Y. 
Kim, S. J. and Kim, D. (1999), "Forced convection in microstructure for electronic equipment cooling", Journal of Heat Transfer, Vol. 121, no.3, pp. 639-645.

Matlab, Version 6.1, (2001), The MathWorks, Inc., Natwick, MA.

Ryu, J. H., Choi, D. H. and Kim, S. J. (2002), "Numerical optimization of the thermal performance of a microchannel heat sink", International Journal of Heat Mass Transfer, Vol. 45 , no. 13 , pp. 2823-2827.

Sabry, M.-N. (2001), "Transverse temperature gradient effect on fin efficiency for microchannel design", Journal of Electronic Packaging, Vol. 123, no. 4, pp. 344-350.

Samalam, V. K. (1989), "Convective heat transfer in microchannels", Journal of Electronic Materials, Vol. 18, no. 5, pp. 611- 617.

Shah, R. K. and London, A. L. (1978), "Laminar flow forced convection in ducts", Advances in Heat Transfer, Supplement 1, Academic Press.

Sobhan, C. B. and Garimella, S. V. (2001), "A comparative analysis of studies on heat transfer and fluid flow in microchannels", Microscale Thermophysical Engineering, Vol. 5, no. 4, pp. 293-311.

Tien, C. L. and Kuo, S. M. (1987), "Analysis of forced convection in microstructures for electronic system cooling", Proc. Int. Symp. Cooling Tech. for Electronic Equipment, Honolulu, HI, pp. 217-226.

Toh, K. C., Chen, X. Y. and Chai, J.C. (2002), "Numerical computation of fluid flow and heat transfer in microchannels", International Journal of Heat Mass Transfer, Vol. 45, no. 2, pp. 5133-5141.

Tuckerman, D. B. and Pease, R. F. (1981), "High-performance heat sinking for VLSI", IEEE Electronic Devices Letters EDL-2, pp. 126-129.

Vafai, K. and Tien, C. L. (1981), "Boundary and inertia effects on flow and heat transfer in porous media", International Journal of Heat Mass Transfer, Vol. 24, no. 2, pp. 195 -203.

Weisberg, A. and Bau, H. H. (1992), "Analysis of microchannels for integrated cooling", International Journal of Heat Mass Transfer, Vol. 35, no. 10, pp. 2465-2474.

Zhao, C. Y. and Lu, T. J. (2002), "Analysis of microchannel heat sinks for electronics cooling", International Journal of Heat Mass Transfer, Vol. 45, no. 24, pp. 4857-4869. 


\section{FIGURE CAPTIONS}

Figure 1. Schematic of a microchannel heat sink.

Figure 2. Computational domain.

Figure 3. Heat flux distribution at the base of the fin.

Figure 4. Temperature profile in a microchannel heat sink. 
Table I. Comparison of thermal resistances.

\begin{tabular}{|c|c|c|c|}
\hline & \multicolumn{3}{|c|}{ Case } \\
\cline { 2 - 4 } & 1 & 2 & 3 \\
\hline$W_{c}(\mu \mathrm{m})$ & 56 & 55 & 50 \\
\hline$W_{w}(\mu \mathrm{m})$ & 44 & 45 & 50 \\
\hline$H_{c}(\mu \mathrm{m})$ & 320 & 287 & 302 \\
\hline $\mathrm{H}(\mu \mathrm{m})$ & 533 & 430 & 458 \\
\hline$\Delta \mathrm{P}(\mathrm{kPa})$ & 103.42 & 117.21 & 213.73 \\
\hline $\mathrm{q} "\left(\mathrm{~W} / \mathrm{cm}^{2}\right)$ & 181 & 277 & 790 \\
\hline $\mathrm{R}_{\exp }\left({ }^{\circ} \mathrm{C} / \mathrm{W}\right)^{*}$ & 0.110 & 0.113 & 0.090 \\
\hline $\mathrm{R}_{\text {num }}\left({ }^{\circ} \mathrm{C} / \mathrm{W}\right)$ & 0.115 & 0.114 & 0.093 \\
\hline
\end{tabular}

Tuckerman and Pease (1981); L = W =1 cm 
Table II. Summary of approximate analytical models.

\begin{tabular}{|c|c|c|c|c|c|c|}
\hline \multirow[t]{2}{*}{ Model } & \multicolumn{2}{|c|}{ Temperature } & \multicolumn{2}{|c|}{$\begin{array}{c}\text { Axial } \\
\text { conduction }\end{array}$} & \multirow[t]{2}{*}{ Governing equations } & \multirow[t]{2}{*}{ Thermal resistance $(\mathbf{R})$} \\
\hline & Fin & Fluid & Fin & Fluid & & \\
\hline 1 & $1-\mathrm{D}$ & $1-\mathrm{D}$ & $\mathrm{X}$ & $x$ & Not used & $\frac{1}{n h L\left(2 \eta_{f} H_{c}+w_{c}\right)}+\frac{1}{\rho_{f} Q C_{p}}$ \\
\hline 2 & $2-D$ & $1-D$ & $\mathrm{X}$ & $X$ & $\begin{array}{l}\frac{d^{2} T}{d y^{2}}-\frac{h P}{k_{s} A_{c}}\left(T-T_{f}(x)\right)=0 \\
\dot{m} C_{p} \frac{d T_{f}(x)}{d x}=q \cdot\left(w_{c}+w_{w}\right)\end{array}$ & $\frac{1}{n h L\left(2 \eta_{f} H_{c}+w_{c}\right)}+\frac{1}{\rho_{f} C_{p} Q}$ \\
\hline 3 & 2-D & $2-D$ & $x$ & $X$ & $\begin{array}{l}k_{s} \frac{\partial^{2} T}{\partial y^{2}}=\frac{2 h}{w_{w}}\left(T-T_{f}(x, y)\right) \\
\rho_{f} C_{p} u_{m} w_{c} \frac{\partial T_{f}}{\partial x}=2 h\left(T-T_{f}(x, y)\right)\end{array}$ & $\frac{J a}{k_{s} \tilde{H}_{c} \beta}\left[(1+L / a)+\frac{1}{3} \beta \tilde{H}_{c}^{2}\right] \frac{1}{(L W)}$ \\
\hline 4 & 2-D & $2-D$ & $\sqrt{ }$ & X & $\begin{array}{l}\nabla^{2} T(x, y, z)=0 \\
\nabla \cdot\left(\vec{V} T_{f}(x, y, z)\right)=\alpha_{f} \nabla^{2} T_{f}(x, y, z)\end{array}$ & $\begin{array}{r}\frac{2 \eta_{f} \alpha}{2 \eta_{f} \alpha+1}\left[\frac{1}{2 n h H_{c} \eta_{f} L}+\frac{1}{\rho_{f} C_{p} Q}+\frac{1}{\left(\rho_{f} C_{p} Q\right)^{2}} \frac{n k_{s} H_{c} w_{w}}{L}\right] \\
(91)\end{array}$ \\
\hline 5 & $1-\mathrm{D}$ & $1-D$ & X & $x$ & $\begin{array}{l}-\frac{d}{d x}\langle p\rangle_{f}+\mu_{f} \frac{d^{2}}{d y^{2}}\langle u\rangle_{f}-\frac{\mu_{f}}{K} \varepsilon\langle u\rangle_{f}=0 \\
k_{s e} \frac{\partial^{2}\langle T\rangle}{\partial y^{2}}=h a\left(\langle T\rangle-\langle T\rangle_{f}\right) \\
\varepsilon \rho_{f} C_{p}\langle u\rangle_{f} \frac{\partial\langle T\rangle_{f}}{\partial x}=h a\left(\langle T\rangle-\langle T\rangle_{f}\right)+k_{f e} \frac{\partial^{2}\langle T\rangle_{f}}{\partial y^{2}}\end{array}$ & $\frac{12 K}{\rho_{f} C_{p} w_{c}^{3} \alpha W \varepsilon^{2} u_{m}}-\frac{\theta_{f, b} H_{c}}{(1-\varepsilon) k_{s} L W}$ \\
\hline
\end{tabular}

(X-not considered, $\sqrt{ }$ - considered) 
Table III. Overall thermal resistances.

\begin{tabular}{|c|c|c|c|}
\hline \multirow{2}{*}{$\begin{array}{c}\text { Thermal } \\
\text { resistance }\end{array}$} & \multicolumn{3}{|c|}{ Case } \\
\cline { 2 - 4 }$\left({ }^{\circ} \mathrm{C} / \mathrm{W}\right)$ & 1 & 2 & 3 \\
\hline $\mathrm{R}_{0, \text { num }}$ & 0.115 & 0.114 & 0.093 \\
\hline $\mathrm{R}_{0, \text { model } 1}$ & 0.112 & 0.112 & 0.091 \\
\hline $\mathrm{R}_{0, \text { model } 2}$ & 0.112 & 0.112 & 0.091 \\
\hline $\mathrm{R}_{0, \text { model } 3}$ & 0.106 & 0.106 & 0.087 \\
\hline $\mathrm{R}_{0, \text { model } 4}$ & 0.106 & 0.106 & 0.087 \\
\hline $\mathrm{R}_{0, \text { model } 5}$ & 0.115 & 0.106 & 0.089 \\
\hline
\end{tabular}


Table IV. Nusselt numbers.

\begin{tabular}{|l|c|c|c|}
\hline \multirow{2}{*}{} & \multicolumn{3}{|c|}{ Case } \\
\cline { 2 - 4 } & 1 & 2 & 3 \\
\hline$N u_{f d}$ & 5.97 & 5.81 & 6.06 \\
\hline$N u$ & 5.60 & 5.55 & 5.85 \\
\hline
\end{tabular}


Table V. Optimal dimensions.

\begin{tabular}{|c|c|c|c|c|}
\hline Model & $\begin{array}{c}W_{c} \\
(\mu \mathrm{m})\end{array}$ & $\begin{array}{c}W_{w} \\
(\mu \mathrm{m})\end{array}$ & $\alpha$ & $\begin{array}{c}\mathrm{R}_{\circ} \\
\left({ }^{\circ} \mathrm{C} / \mathrm{W}\right)\end{array}$ \\
\hline 1 & 64 & 18 & 6.25 & 0.0965 \\
\hline 2 & 65 & 19 & 6.15 & 0.0965 \\
\hline 3 & 65 & 24 & 6.15 & 0.0973 \\
\hline 4 & 61 & 16 & 6.56 & 0.0907 \\
\hline 5 & 64 & 27 & 6.25 & 0.1072 \\
\hline
\end{tabular}




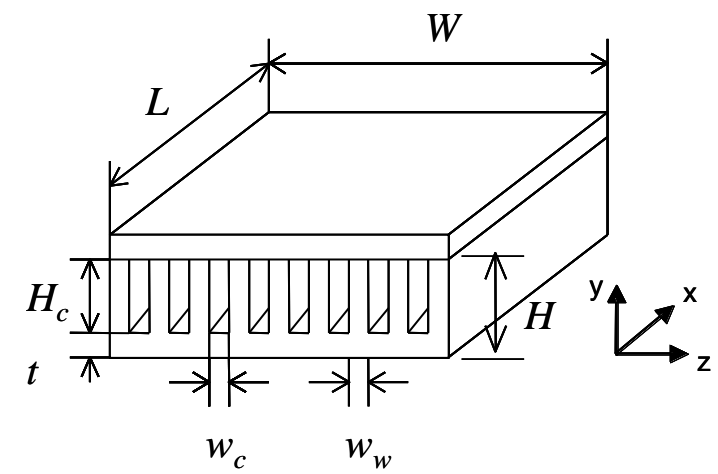

Fig. 1 Schematic of a microchannel heat sink.

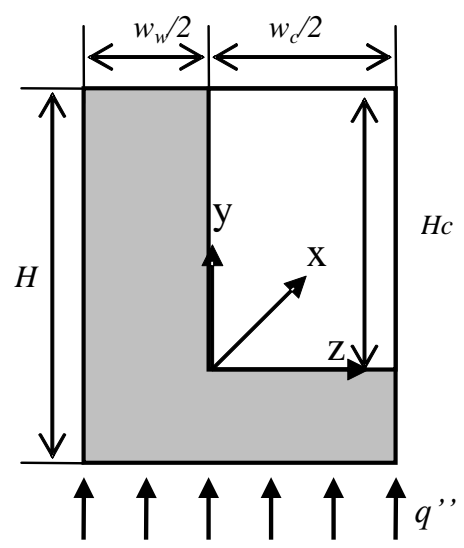

Fig. 2 Computational domain. 


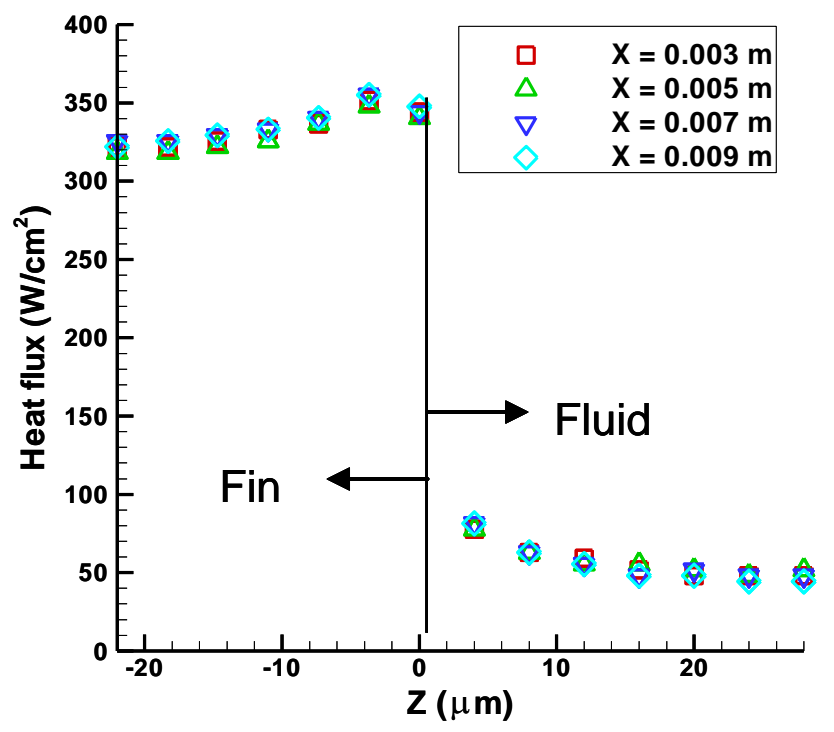

Fig. 3 Heat flux distribution at the base of the fin.

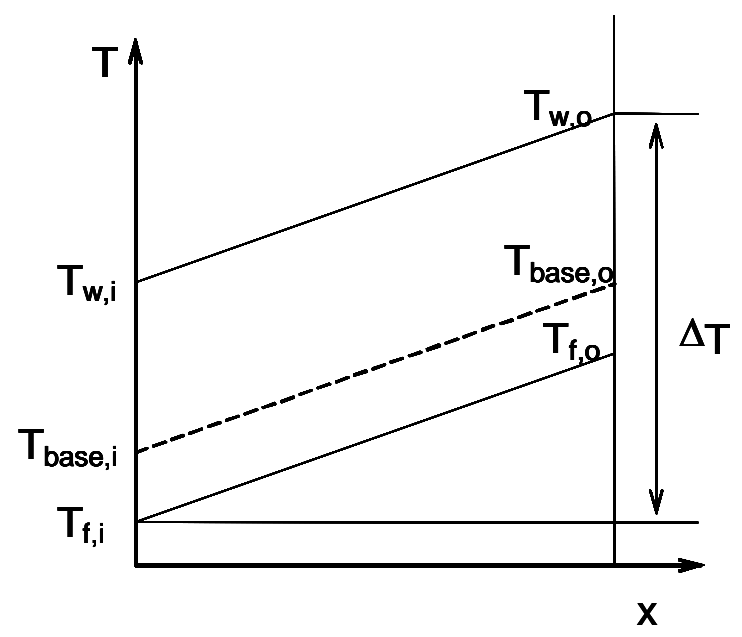

Fig. 4 Temperature profile in a microchannel heat sink. 